\title{
A Tangible Approach to Time Management
}

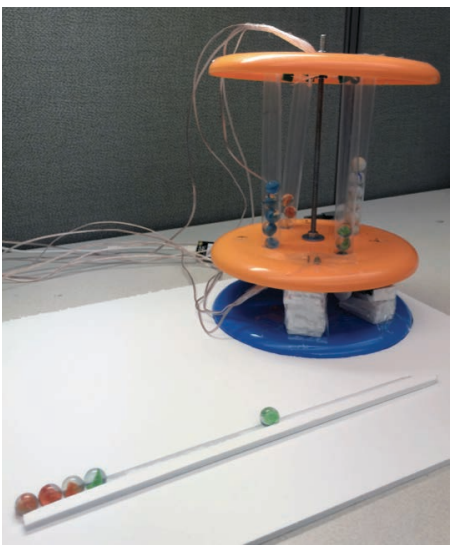

Figure 1. Time Machine. An early prototype of an ambient display and tangible interface for time management. The device utilizes colored glass marbles to

represent units of time

(Pomodoros) and clear cylinders as tasks. Phidgets [4] enable

timed release of marbles into the elapsed-time rail from the active task cylinder. Task switching is supported by rotating the top (orange) portion of the device. Physical switches are used to determine time allocation on task columns and detect task switches.

\section{Ryan Ahmed}

Indiana University, IUPUI

535 W Michigan Avenue

Indianapolis, IN USA 46202

ahmedr@iupui.edu

\section{Alex Chambers}

Indiana University, IUPUI

535 W Michigan Avenue

Indianapolis, IN USA 46202

chambera@iupui.edu

\section{Michael Frontz}

Indiana University, IUPUI

535 W Michigan Avenue

Indianapolis, IN USA 46202

mfrontz@iupui.edu

\section{Stephen Voida}

Indiana University, IUPUI

535 W Michigan Avenue

Indianapolis, IN USA 46202

svoida@iupui.edu
Permission to make digital or hard copies of part or all of this work for personal or classroom use is granted without fee provided that copies are not made or distributed for profit or commercial advantage and that copies bear this notice and the full citation on the first page. Copyrights for third-party components of this work must be honored. For all other uses, contact the Owner/Au this

Copyright is held by the owner/author(s).

UbiComp '14 Adjunct, September 13-17, 2014, Seattle, WA, USA ACM $978-1-4503-3047-3 / 14 / 09$

http://dx.doi.org/10.1145/2638728.2638794

\begin{abstract}
Information work generally occurs within a multitasking environment with attention focused on the computer screen, constant task switching and frequent interruptions. In this environment, software-based task management techniques may blend in too much to be optimally effective. The Time Machine is proposed as a physical interface distinctly separated from the task environment with real-world manifestations of arbitrary concepts of tasks and time, while providing constant visibility of status through an ambient display for selfreflection. The Time Machine aims to promote distributed cognition and utilize the stage-based model of personal informatics and the Pomodoro technique toward productive and enjoyable task management.
\end{abstract}

\section{Author Keywords}

Tangible user interface; personal informatics; ambient display; Phidgets; distributed cognition

\section{ACM Classification Keywords}

H.5.m. Information interfaces and presentation (e.g., $\mathrm{HCI}$ ): Miscellaneous

\section{Introduction}

Because time is not physical, the human perception of time is subjective: the passage of time can seem slower or faster depending on factors like task 


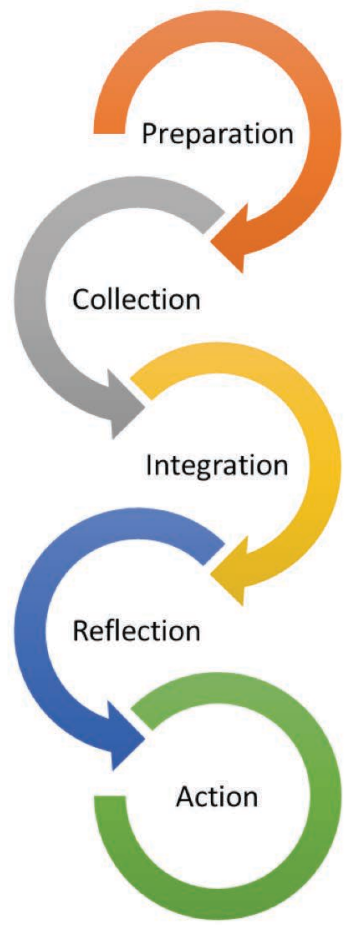

Figure 2. The stage-based mode of personal informatics systems (after [6]). engagement. For example, when deeply engaged in a task to the point of experiencing flow, a person's subjective experience of time is typically altered [3]. Thus, people have historically relied on visual or auditory signals to estimate, determine, or track time. Initially, these time signals were derived from nature: the position of the sun in the sky or the sound of a rooster in the morning. Eventually, these time signals became technology-based. Many people now rely on displays, or signals, of time that derive from our world of pervasive devices: digital time displayed on a device screen, a notification sound from a calendar application.

Time management can be challenging, especially given the pervasiveness of multitasking. Most existing software do not provide comprehensive support for time-management, including task visibility, reminders to return to a task after interruption, and a sense of progress toward completion of multiple ongoing tasks. Software can also be interruptive, since both the time management and primary work tools share the same task environment, causing additional context switches.

Although researchers previously have studied mechanisms and techniques for personal time management, there appears to be a lack of published research that addresses time or task management using tangible or ambient user interfaces. One notable exception is the Japanese Garden [7], an interface that also used tangible representations of tasks (stones), but primarily as a communicative medium about availability among a group of distributed collaborators.

\section{Design Rationale}

The device's role as an ambient display is intended to aid memory and reduce cognitive load. Drawing on the theory of distributed cognition [5], we utilize representational physical artifacts to provide a tangible interface for task planning, aural cues for time passage, and an ambient, glanceable display to convey status. Marbles are used as metaphorical representations of blocks of time. They enable users to visualize work progress and task load as they flow from the task cylinders onto a collection rail through an audible, timed-release mechanism.

Each marble represents 30 minutes of time. These 30minute time blocks are based on the $25+5$ minute Pomodoro Technique of time management [1], devised to minimize interruptions. The core theory revolves around an iterative process involving 25 minutes of concentrated focus followed by a 5-minute break.

The design principle of the Time Machine follows the stage-based model of personal informatics systems proposed by Li, Dey and Forlizzi [6]. The iterative model includes five main stages of preparation, collection, integration, reflection, and action (Figure 2). What we hope to achieve with the Time Machine is to alleviate the need for a user to navigate these stages for time management using solely their own memory and knowledge. Our device is focused on supporting the users from the preparation stage through the reflection stage, which we will highlight in the following discussion of our initial system implementation.

\section{Prototype Implementation}

Our design utilizes marbles as a physical object metaphor for representing units of time (Figures 1 and 3). This design is partly inspired by Bishop's Marble Answering Machine [2]. However, in addition, marbles also offer several specific physical advantages: 


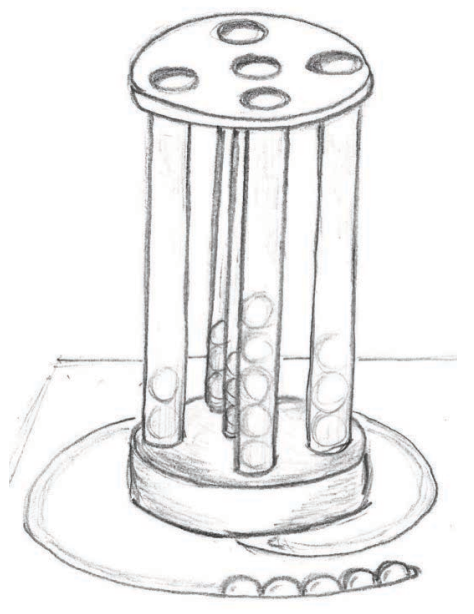

Figure 3. A conceptual drawing of a more polished instantiation of the Time Machine device, which we hope to produce for future demonstrations and deployment testing.
- Due to their size and shape, marbles are easy (and pleasing) to grasp and manipulate.

- The symmetrical shape allows marbles to be placed easily, without regard to orientation.

- The spherical shape allows marbles to easily move and roll through the various sections of the device representing planned time and elapsed time.

- Marbles come in a variety of colors, allowing each task to be tracked by marbles of a unique color.

\section{Preparation Stage}

The preparation stage begins when a user assigns each planned task to a cylinder and fills it with colored marbles matching his or her estimation of required time. Our prototype has 4 task cylinders -3 dedicated to tasks and a 4th to account for breaks. By using switches, the Time Machine can sense the quantity of marbles (time units) added to each particular task, track elapsed time for the current task marble, and track the number of marbles remaining for each task. If all of the marbles for a particular task have been used, the device notifies the user (optionally, through an ambient sound) to either switch to a new task or to add additional marbles to the empty task cylinder.

\section{Collection and Integration Stages}

Following the Pomodoro technique, at the conclusion of each block of time (25 minutes), a servo motor drops one marble from the current task's cylinder and a new 25-minute block starts countdown after a 5-minute break. Each time the user hears a marble drop, he or she will have 5 minutes to take a short break away from the task and, potentially, to switch working tasks. Once the 5-minute break window has passed, the system "locks" in the next activity and resets the timer for another 25 minutes.
The Reflection Stage

Since time elapses in a linear fashion and users may switch between tasks during the course of a day, the "elapsed" marbles roll into a track below the storage cylinders. The fact that each task is represented by a stack of marbles of a particular color allows a user to reflect on how they spent their time. The color, quantity, and order of the marbles suggests which task was worked on at a given time, how much time was spent on that task before switching to a different task, how frequently task switching occurred, and when breaks occurred. In short, the reflection "row" serves as a visual graph of the how the user spent his or her time during the day.

\section{The Action Stage}

During the action stage, the user decides what to do based on his or her reflection. The action could be simply to better understand what tasks (or types of tasks) require more time or to draw attention to unproductive task-switching behaviors (i.e., "thrashing").

We expect that this system will help users to more fluidly transition among these stages. At the outset of a typical day, users begin in the preparation stage, using the Time Machine to establish task goals and estimate the time required for each. As the day progresses, the device passively collects data about the user's task progress (collection stage) and provides a glanceable display. This display prompts the user to transition to the integration stage by providing a visible reminder of interrupted tasks that require resumption and allowing the user to prioritize or amend his or her initial time estimates. When users move on to the action stage, any visible shortages in allocated time (low marble 
counts) prompt him or her to consciously set aside more time for high-priority tasks, facilitating a transition back to the preparation stage.

Our initial prototype uses a plastic and Foamcore structure with glass marbles and transparent cylinders (Figure 1). Functionality and sensing is handled via a Phidgets InterfaceKit connected to a laptop USB port, and the marbles are dropped by a container connected to a PhidgetAdvancedServo device [4]. The controller software is written in Java.

\section{The Challenge of Representing Time}

Representing time in a tangible interface is a research challenge, as people can experience time at a variety of granularities whereas physical manifestations of time need to represent fixed, integral units. To address this challenge, we implemented the concept of

"commitment" to a Pomodoro. When the user "locks" in their intention to work on a particular task, the system retrieves and stores a marble of that task's color, even if the user switches to another activity. This acts as passive encouragement to follow through on the completion of a task, one of the hallmarks of the Pomodoro approach.

\section{Conclusion and Future Work}

We have introduced a novel approach to time management using a device embodying characteristics of both an ambient display and a tangible user interface. Our system seeks to minimize distraction within the task environment, help manage interruptions, improve distributed cognition and facilitate a user's movement through multiple stages of self-reflection about personal time management. An evaluation of the functional prototype will be an important next step in the research to explore user perceptions of the system and to understand how daily use of the Time Machine might affect behavior change. Because the system is implemented with a Phidgets interface, we will be able to collect data to enable a comparative analysis of users' multitasking performance based on interruption count, task resumption and completion counts with and without Time Machine, and to carry out a comparison of estimated versus actual time spent on planned tasks.

\section{Acknowledgements}

We thank all of our colleagues in the IUPUI Department of Human-Centered Computing for their constructive feedback.

\section{References}

[1] Cirillo, F. The Pomodoro Technique. XPLabs Technical Report, version 1.3 (June 15, 2007), http://www.tecnicadelpomodoro.it

[2] Crampton Smith, G. (1995). The hand that rocks the cradle. I.D. magazine, (May-June 1995), 60-65.

[3] Csikszentmihalyi, M. Beyond Boredom and Anxiety. Jossey-Bass, San Francisco, 1975.

[4] Greenberg, S. and Fitchett, C. Phidgets: Easy development of physical interfaces through physical widgets. In Proc. UIST 2001. ACM Press (2001), 209-218.

[5] Hollan, J., Hutchins, E., and Kirsh, D. Distributed cognition: Toward a new foundation for humancomputer interaction research. ACM Trans. CHI 7, 2 (2000), 174-196.

[6] Li, I., Dey, A., and Forlizzi, J. A stage-based mode of personal informatics systems. In Proc. CHI 2010. ACM Press (2010), 557-566.

[7] Yuzawa, H. and Mark, G. The Japanese Garden: Task awareness for collaborative multitasking. In Proc. GROUP 2010. ACM Press (2010), 253-262. 